\title{
Correlation of symptoms of premenstrual syndrome in Indian set-up
}

\author{
Preeti Garg ${ }^{1}$, Prithpal S Matreja ${ }^{2, ~}$, Prem P Khosla ${ }^{3}$, Lovepreet Kaur ${ }^{4}$, Praveen Mohan \\ ${ }^{1}$ Department of Pharmacology, Gian Sagar Medical College and Hospital, Village Ram Nagar, District Patiala, Punjab, India-140601 \\ ${ }^{2}$ Department of Pharmacology, Gian Sagar Medical College and Hospital, Village Ram Nagar, Tehsil Rajpura, District Patiala, Punjab, \\ India-140601 \\ ${ }^{3}$ Department of Pharmacology, Subharti Medical College and Hospital, Meerut, Uttar Pradesh, India-250002 \\ ${ }^{4}$ Gian Sagar Medical College and Hospital, Village Ram Nagar, District Patiala, Punjab, India-140601 \\ ${ }^{5}$ Department of Obstetrics and Gynecology, MM Institute of Medical Science and Research, Mullana, Harayana, India - 133207
}

\section{Email address:}

drpsmatreja@yahoo.co.in (P. S. Matreja)

\section{To cite this article:}

Preeti Garg, Prithpal S Matreja, Prem P Khosla, Lovepreet Kaur, Praveen Mohan. Correlation of Symptoms of Premenstrual Syndrome in Indian Set-Up. American Journal of Health Research. Vol. 2, No. 3, 2014, pp. 102-105. doi: 10.11648/j.ajhr.20140203.15

\begin{abstract}
Premenstrual symptoms (PMS) occur very commonly and cause a negative impact on the overall quality of life of the women. These symptoms cause distress, depression and strain interpersonal relationships. Little is known about PMS in India hence, this study was designed to know the prevalence of these symptoms and any correlation between them in adult Indian population. This survey was conducted in 150 healthy volunteers in the later half of the menstrual cycle. A predesigned questionnaire regarding PMS was filled. The study was approved by the Institutional Ethics Committee and only those participants were enrolled who were willing to give written informed consent. A total of 148 females were enrolled in the study and divided into 2 groups. All the parameters were comparable except for a significant $(\mathrm{p}<0.05)$ difference in higher complaints of insomnia in group 1. PMS A which considered symptoms of anxiety, irritability, mood swings and nervousness. PMS C included symptoms like increase in appetite, headache, fatigue, dizziness and palpitation. PMS D included symptoms of depression, crying, forgetfulness, confusion and insomnia. PMS H included symptoms of fluid retention, increase in weight, and swelling of extremities, breast tenderness and abdominal bloating. PMS A was significantly correlated with PMS C, PMS D, backache and abdominal bloating in both group 1 and 2. PMS C was significantly correlated with PMS A, PMS D, PMS H, insomnia and generalized aches in both group 1 and 2. PMS D was significantly correlated with PMS A, PMS C, PMS H in both group 1 and 2. PMS H was significantly correlated with PMS $\mathrm{C}$ and PMS D in both group 1 and 2. PMS H has significant correlation with PMS A and insomnia in group 1 and age in group 2. Our study shows premenstrual symptoms are very common and distressing and there is a significant correlation between the various physical, emotional and psychological symptoms.
\end{abstract}

Keywords: Premenstrual Syndrome, Menstrual Cycle, Questionnaire, Physical, Emotional, Psychological

\section{Introduction}

Premenstrual syndrome (PMS) has been defined by National Institute of Mental Health as: the cyclic occurrence of symptoms that are of sufficient severity to interfere with some aspects of life and which appear with consistent and predictable relationship to menses (1).

PMS is among the most common health problems reported by reproductive age women, $70-90 \%$ of women complain of recurrent PMS but not all of them suffer from PMS. About two-third of the patients referred to PMS Clinic by general practitioners do not suffer from PMS and this over-diagnosis may be due to exacerbation of many functional psychiatric disorders during the premenstrual phase of the cycle (2)

PMS consists of emotional and behavioral symptom like anxiety, depression, crying spells, mood swings, irritability, appetite changes and food cravings, insomnia, social withdrawal, poor concentration. Physical signs and symptoms may include bodyaches, headache, fatigue, weight gain, abdominal bloating, and breast tenderness. (3)

Although the list of potential signs and symptoms is long, most women with premenstrual syndrome experience only a few of these. These symptoms occur in a predictable and 
cyclical manner, appearing in the luteal phase of the menstrual cycle and regress with the start of menses. (4)

Among the women who suffer from PMS, mood and behavioral symptoms such as irritability, tension and labile mood dominate. Somatic complaints, including breast tenderness and bloating can also prove disruptive to women's overall functioning and quality of life. (5)

PMS increases distress, anxiety and impairs functioning, reducing work productivity (6), these physical and emotional symptoms influence women's daily activity, personal relationships and work. (7) Regardless of their severity, though, the signs and symptoms disappear, for most women, as the menstrual period begins. A few individuals with severe symptoms are prone to suffer from premenstrual dysphoric disorder (PMDD). A number of women with severe PMS may have an underlying psychiatric disorder. (4)

The exact causes of PMS are not clearly understood, but a number of factors have been implicated like neurotransmitters, prostaglandins, diet, lifestyle and intrinsic hormonal fluctuations of the menstrual cycle. $(8,9,10)$. Diagnostic criteria and management approaches are also varied. A large number of drugs are being investigated for this condition. (11).

Up to $25 \%$ of women may warrant treatment for the distress or impaired functioning associated with PMS. (12). Both physical and mental premenstrual symptoms have significant impact on quality of life $(13,14)$. Symptoms of PMS can be relieved or reduced through lifestyle modifications, such as dietary changes, yoga, meditation and exercise, and drug therapy with hormonal or psychotropic agents. $(15,16)$

Current treatment options include lifestyle changes, non steroidal anti-inflammatory drugs (NSAIDS), selective serotonin reuptake inhibitors (SSRI's) and ovulation suppression (e.g. with oral contraceptives) (10). Long acting analogues of gonadotropin releasing hormone or oestradiol effectively reduce symptoms .Anxiolytics and spironolactone can be used as supportive care to relieve symptoms (11). Cognitive therapy, chasteberry and calcium carbonate may be helpful. (17)

Many women with a troublesome premenstrual disorder go undiagnosed, either because they do not report their symptoms to a clinician or because the clinician has difficulty diagnosing the disorder. (18). There is a considerable variation in the estimated prevalence of PMS because of lack of universally accepted diagnostic criteria and differences in the interpretation of premenstrual symptoms. Little is known about PMS in India hence, this study was designed to know the prevalence of these symptoms and any correlation between them in adult Indian population

\section{Material and Methods}

Study Design: 166 subjects in the premenstrual phase of the cycle coming as attendants in the Obstetrics and
Gynecology outpatients department of Gian Sagar Medical College and Hospital, Patiala from April 2011 to January 2012 were enrolled in the study. The study was commenced only after obtaining the permission from the Institutional Ethics Committee.

Participants in the age group of 18-45 years with regular, normal menstrual cycle ranging between 21-35 days and willing to give written informed consent were included in the study.

All the participants with concurrent psychiatric illness, use of drug/medication, taking any prescribed medication for PMS, receiving psychotherapy, suffering from serious medical illness were excluded from the study. Participants who were pregnant or lactating were also excluded from the study.

Procedure: Survey of symptoms during later half of the menstrual cycle. The data was analyzed in a descriptive manner and compared among the study sample. The patients were divided into two groups depending on the day of the cycle on which they filled the questionnaire. Group 1 consists of patients in day 23-25 of the menstrual cycle while Group 2 consists of the patients in day 26-29 of the menstrual cycle.

A designed questionnaire based on the symptoms of PMS was filled. PMS A which considered symptoms of anxiety, irritability, mood swings and nervousness. PMS C included symptoms like increase in appetite, headache, fatigue, dizziness and palpitation. PMS D included symptoms of depression, crying, forgetfulness, confusion and insomnia. PMS H included symptoms of fluid retention, increase in weight, and swelling of extremities, breast tenderness and abdominal bloating. (19)

\section{Statistical Analysis}

The data was presented as mean \pm standard error (Mean \pm SE). The statistical software SPSS (version 16) was used for data analysis. For comparison of continuous variables between groups Student's t-test was used. Correlations between the variables were examined using the Pearson correlation coefficients. A $\mathrm{p}<0.05$ was considered statistically significant.

\section{Results}

A total of 166 females in 23-29 day of menstrual cycle were screened for the enrollment in the study. A total of 18 females did not give written consent for enrollment in the study and hence were excluded from the study. A total of 148 females were enrolled in the study and divided into 2 groups. Group 1 included females who reported on 23-25 day of menstrual cycle and Group 2 included females who reported on 26-29 day of menstrual cycle. The demographic profiles of participants in both the groups are shown in Table 1. All the parameters were comparable except for a significant $(\mathrm{p}<0.05)$ difference in higher complaints of insomnia in group 1. The average age of 
participants in Group 1 was $26.33 \pm 0.79$ years and $27.67 \pm$ 0.79 years in participants of group 2. An estimate of correlation for PMS A and PMS C with other variables along with their significant levels among the participants in both groups is shown in Table 2. PMS A has significant $(\mathrm{p}<0.05)$ correlation with PMS C, PMS D, backache and abdominal bloating in both group 1 and 2. PMS A is significantly correlated with PMS $\mathrm{H}$ and insomnia in group1 whereas, PMS A shows significant correlation with generalized aches in group 2.PMS $\mathrm{C}$ has significant $(\mathrm{p}<0.05)$ correlation with PMS A, PMS D, PMS H , insomnia and generalized aches in both group 1 and 2 and has significant correlation with backache and abdominal bloating in group 2. An estimate of correlation for PMS D and PMS H with other variables along with their significant levels among the participants in both groups is shown in Table 3. PMS D has significant $(\mathrm{p}<0.05)$ correlation with PMS A, PMS C, PMS H in both group 1 and 2 . PMS D has significant $(\mathrm{p}<0.05)$ correlation with insomnia in group
1 whereas, PMS D shows significant correlation with backache, abdominal bloating and generalized aches in group 2. PMS $H$ has significant $(\mathrm{p}<0.05)$ correlation with PMS $\mathrm{C}$ and PMS D in both group 1 and 2. PMS $\mathrm{H}$ has significant correlation with PMS A and insomnia in group 1 and age in group 2

Table 1. Demographic Profile (Mean \pm SE) of participants in both groups.

\begin{tabular}{llll}
\hline Characteristics & Group 1(n=61) & Group 2(n=87) & p value \\
\hline Age (years) & $26.33 \pm 0.79$ & $27.67 \pm 0.79$ & $0.24 \#$ \\
PMS A & $2.30 \pm 0.32$ & $1.84 \pm 0.22$ & $0.22 \#$ \\
PMS C & $1.33 \pm 0.22$ & $1.46 \pm 0.18$ & $0.64 \#$ \\
PMS D & $0.75 \pm 0.22$ & $0.41 \pm 0.10$ & $0.11 \#$ \\
PMS H & $1.36 \pm 0.30$ & $1.33 \pm 0.22$ & $0.94 \#$ \\
Backache & $1.20 \pm 0.13$ & $1.28 \pm 0.12$ & $0.66 \#$ \\
Insomnia & $0.21 \pm 0.07$ & $0.05 \pm 0.02$ & $0.02 * \#$ \\
Abdominal Bloating & $0.82 \pm 0.13$ & $1.10 \pm 0.11$ & $0.10 \#$ \\
Generalized Aches & $0.85 \pm 0.12$ & $0.90 \pm 0.10$ & $0.78 \#$ \\
\hline
\end{tabular}

${ }^{*} \mathrm{p}<0.05$ as compared to other group

Table 2. Correlation coefficient for PMS A and PMS C with other variables in both groups.

\begin{tabular}{|c|c|c|c|c|c|c|c|c|}
\hline \multirow{3}{*}{ Characteristics } & \multicolumn{4}{|c|}{ PMS A } & \multicolumn{4}{|c|}{ PMS C } \\
\hline & \multicolumn{2}{|c|}{ Group 1} & \multicolumn{2}{|c|}{ Group 2} & \multicolumn{2}{|c|}{ Group 1} & \multicolumn{2}{|c|}{ Group 2} \\
\hline & $\mathbf{r}$ & $\mathbf{p}$ & $\mathbf{r}$ & $\mathbf{p}$ & $\mathbf{r}$ & $\mathbf{p}$ & $\mathbf{r}$ & $\mathbf{p}$ \\
\hline Age & 0.16 & 0.23 & 0.16 & 0.29 & 0.03 & 0.81 & 0.03 & 0.78 \\
\hline PMS A & 1 & - & 1 & & 0.44 & $<0.05$ & 0.57 & $<0.05$ \\
\hline PMS C & 0.44 & $<0.05$ & 0.57 & $<0.05$ & 1 & & 1 & \\
\hline PMS D & 0.55 & $<0.05$ & 0.51 & $<0.05$ & 0.65 & $<0.05$ & 057 & $<0.05$ \\
\hline PMS H & 0.47 & $<0.05$ & 0.12 & 0.26 & 0.46 & $<0.05$ & $0.27^{*}$ & $<0.05$ \\
\hline Backache & 0.45 & $<0.05$ & 0.32 & $<0.05$ & 0.24 & 0.06 & 0.45 & $<0.05$ \\
\hline Insomnia & 0.37 & $<0.05$ & 0.13 & 0.24 & 0.71 & $<0.05$ & 0.37 & $<0.05$ \\
\hline Abdominal Bloating & 0.27 & $<0.05$ & 0.28 & $<0.05$ & 0.23 & 0.07 & 0.38 & $<0.05$ \\
\hline Generalized Aches & 0.15 & 0.24 & 0.44 & $<0.05$ & 0.38 & $<0.05$ & 0.47 & $<0.05$ \\
\hline
\end{tabular}

Table 3. Correlation coefficient for PMS D and PMS H with other variables in both groups.

\begin{tabular}{|c|c|c|c|c|c|c|c|c|}
\hline \multirow{3}{*}{ Characteristics } & \multicolumn{4}{|c|}{ PMS D } & \multicolumn{4}{|c|}{ PMS H } \\
\hline & \multicolumn{2}{|c|}{ Group 1} & \multicolumn{2}{|c|}{ Group 2} & \multicolumn{2}{|c|}{ Group 1} & \multicolumn{2}{|c|}{ Group 2} \\
\hline & $\mathbf{r}$ & $\mathbf{p}$ & $\mathbf{r}$ & $\mathbf{p}$ & $\mathbf{r}$ & $\mathbf{p}$ & $\mathbf{r}$ & $\mathbf{p}$ \\
\hline Age & 0.18 & 0.16 & 0.07 & 0.53 & 0.07 & 0.59 & 0.28 & $<0.05$ \\
\hline PMS A & 0.55 & $<0.05$ & 0.51 & $<0.05$ & 0.47 & $<0.05$ & 0.12 & 0.26 \\
\hline PMS C & 0.65 & $<0.05$ & 0.57 & $<0.05$ & 0.46 & $<0.05$ & 0.27 & $<0.05$ \\
\hline PMS D & 1 & & 1 & & 0.54 & $<0.05$ & 0.34 & $<0.05$ \\
\hline PMS H & 0.54 & $<0.05$ & 0.34 & $<0.05$ & 1 & & 1 & \\
\hline Backache & 0.17 & 0.18 & 0.34 & $<0.05$ & 0.25 & 0.051 & -0.04 & 0.70 \\
\hline Insomnia & 0.77 & $<0.05$ & 0.14 & 0.18 & 0.47 & $<0.05$ & -0.09 & 0.40 \\
\hline Abdominal Bloating & 0.24 & 0.06 & 0.34 & $<0.05$ & 0.14 & 0.30 & 0.17 & 0.12 \\
\hline Generalized Aches & 0.13 & 0.30 & 0.40 & $<0.05$ & 0.17 & 0.19 & 0.06 & 0.61 \\
\hline
\end{tabular}

\section{Discussion}

The cyclic recurrence of distressing emotional, physical and behavioral symptoms not due to organic or underlying psychiatric disease in the luteal phase of the menstrual cycle which subsides with the onset of menstruation comprises of the premenstrual syndrome. The severity of these symptoms varies as is judged according to the interference with activities of daily life and interpersonal relationships. $(13,14)$

The results of our study showed that a total of 148 females were enrolled in the study and divided into 2 groups. All the parameters were comparable except for a significant difference in higher complaints of insomnia in group 1. PMS A was significantly correlated with PMS C, PMS D, backache and abdominal bloating in both group 1 and 2. PMS C was significantly correlated with PMS A, PMS D, PMS H , insomnia and generalized aches in both group 1 and 2. PMS D was significantly correlated with PMS A, PMS C, PMS H in both group 1 and 2. PMS $\mathrm{H}$ was significantly correlated with PMS C and PMS D in both group 1 and 2. PMS H has significant correlation with PMS A and insomnia in group 1 and age in group 2

A study conducted by Rudolf et.al. showed that variability of premenstrual symptoms is higher in the menstrual and 
premenstrual phase as compared to the intermenstrual phase, the results of our study showed that during the premenstrual phase all the patients in both groups had significant symptoms which were correlated in the group. (20)

Another study showed cramps, backache, fatigue and tension to be most prevalent during the menstrual period while weight gain, painful breasts, swelling, irritability, mood swings and depression were more prevalent in the premenstrual phase whereas our study showed a high prevalence of insomnia, and generalized aches also in the premenstrual phase of the cycle in one of the groups. (21)

In a study by Meadan etal, all the symptoms were found to peak on the first day of the menstruation. The results of our study showed the incidence of all these symptoms during the last week of the menstrual cycle. (22)

In our study, PMS C, PMS, and PMS $\mathrm{H}$ showed significant correlation with each other in both the groups i.e. on all days from day 23 to day 29 of the cycle this shows that these symptoms appear frequently during the premenstrual period and are correlated with each other significantly.

There are certain limitations to our study; firstly, more number of participants could make the results more significant. Secondly, this is a study done at a point of time, if it was conducted over a period of time with follow-up and an intervention could have been done in participants with severe symptoms.

In conclusion, our study shows premenstrual symptoms are very common and distressing; there is a significant correlation between the various physical, emotional and psychological symptoms

\section{References}

[1] Endicott J, Halbreich U, Schact S, Nee, J. Premenstrual changes and affective disorders. Psychosom Med 1981; 43: 519-29

[2] Grosz HJ. Correlates of Premenstrual syndrome. Am J Psychiatry Nov 1988; 145: 1482.

[3] Dennerstein L, Lehert P, Heinemann K. Global study of women's experiences of premenstrual symptoms and their effects on daily life. Menopause Int 2011; 17: 88-95.

[4] Diseases and Conditions. Premenstrual Syndrome (PMS). Available at url. http://www.mayoclinic.org/diseasesconditions/premenstrual-syndrome/basics/symptoms/con20020003. (Last Accessed on 11th April, 2014).

[5] Yonkers KA, O'Brien PM, Eriksson E. Premenstrual syndrome. Lancet 2008; 371: 1200-10.

[6] Tkachenko LV, Kurushina OV, Atagadzheiva MS. The quality of life in women suffering from premenstrual syndrome. Probl Sotsialnoi Gig Zdravookhranenniinai Istor Med 2010; 2: 13-6.
[7] Teixeira AL, Oliveira ÉC, Dias MR. Relationship between the level of physical activity and premenstrual syndrome incidence. Rev Bras Ginecol Obstet 2013; 35: 210-4.

[8] Myint TH, Edessa OG, Sawhsarkapaw. Premenstrual syndrome among female university students in Thailand. AU JT 2006; 9: 158-62.

[9] Rasheed P, Al-soweilem LS. Prevalence and predictors of premenstrual syndrome among college-aged women in Saudi Arabia. Ann Saudi Med 2003; 6: 381-7.

[10] Clayton AH. Symptoms related to the menstrual cycle: diagnosis, prevalence and treatment. J Psychiatr Pract 2008; 14: 13-21.

[11] Jarvis CI, Lynch AM, Morin AK. Management strategies for premenstrual syndrome/ premenstrual dysphoric disorder. Ann Pharmacother 2008; 42: 967-78.

[12] Freeman EW. Therapeutic management of premenstrual syndrome. Expert Opin Pharmacother 2010; 11:2879-89.

[13] Dennerstein L, Lehert P, Bäckström TC, Heinemann K. The effect of premenstrual symptoms on activities of daily life. Fertil Steril 2010; 94: 1059-64.

[14] Dennerstein L, Lehert P, Keung LS, Pal SA, Choi D. Asian study of effects of premenstrual symptoms on activities of daily life. Menopause Int 2010; 16: 146-51

[15] Frackiewicz EJ, Shiovitz TM. Evaluation and management of premenstrual syndrome and premenstrual dysphoric disorder. J Am Pharm Assoc (Wash) 2001; 41: 437-47.

[16] Pearlstein T, Steiner M. Premenstrual dysphoric disorder: burden of illness and treatment update. J Psychiatry Neurosci 2008; 33: 291-301

[17] Zaafrane F, Faleh R, Melki W, Sakouhi M, Gaha L. An overview of premenstrual syndrome. J Gynecol Obstet Biol Reprod (Paris) 2007; 36: 642-52.

[18] Futterman LA, Rapkin AJ. Diagnosis of premenstrual disorders. J Reprod Med 2006; 51(4 Suppl): 349-58.

[19] Wallace K. Premenstrual Syndrome Questionnaire. Available at url. http://www.wallacehealth.com/wpcontent/uploads/2011/10/PMS-Questionnaire.pdf. (Last Accessed on 11th April, 2014).

[20] Moos RH, Kopell BS, Melges FT, Yalom ID, Lunde DT, Clayton RB, et.al. Fluctuations in symptoms and moods during the menstrual cycle. J Psychosom Res 1969; 13: 3744.

[21] Woods NF, Most A, Dery GK. Prevalence of perimenstrual symptoms. Am J Public Health 1982; 72: 1257-64.

[22] Maeden PM, Hartlage SA, Cook-Karr J. Timing and severity of symptoms associated with the menstrual cycle in a community-based sample in the Midwestern United States. Psychiatry Res. 2005; 134: 27-36. 